\title{
MONADIC DEFINABILITY OF ORDINALS
}

\author{
ITAY NEEMAN
}

\begin{abstract}
We identify precisely which singular ordinals are definable by monadic second order formulae over the ordinals, assuming knowledge of the definable regular cardinals.
\end{abstract}

In the paper Neeman [4] the author defines a class of finite state automata acting on transfinite sequences, connects these automata with monadic second order truth over the ordinals, and uses the connection to show that $\aleph_{\omega}$ is not definable by a monadic second order formula over $(\mathrm{ON} ;<)$, and in fact no singular cardinal is definable. This can be viewed as a "negative" result, but it turns out that the same tools can be used to produce some positive results. Here we use the connection between automata and monadic truth to show that if an ordinal $\theta>0$ is definable then:

1. $\operatorname{cof}(\theta)$ is definable.

2. There are definable ordinals $\delta, \gamma<\theta$ so that $\theta=\delta+\gamma \cdot \operatorname{cof}(\theta)$.

This, and the observation that the set of definable ordinals is closed under ordinal addition and multiplication, leads to a complete characterization of the definable singular ordinals in term of the definable regular cardinals: an ordinal is definable by a monadic formula iff it can be obtained from definable regular cardinals using ordinal addition and multiplication. (The results of Magidor [3] strongly suggest that the question of which regular cardinals are definable is independent of ZFC.)

In light of the fact that the definability of $\theta$ implies the definability of $\operatorname{cof}(\theta)$, it is tempting to imagine that, for all $\theta, \operatorname{cof}(\theta)$ is definable with parameter $\theta$. Similarly, in light of the closure of the set of definable ordinals under addition and multiplication, it is tempting to imagine that $\alpha+\beta$ and $\alpha \cdot \beta$ are definable with parameters $\alpha$ and $\beta$. We end the paper with a precise analysis of monadic definability with parameters, showing that both these fantasies are false.

$\S 1$. Preliminaries. For a function $t: \delta \rightarrow S$, where $\delta$ is an ordinal and $S$ a set, define $\operatorname{cf}(t)=\{b \in S \mid$ the set $\{\xi \mid t(\xi)=b\}$ is cofinal in $\delta\}$.

Definition 1.1. Let $S$ be a set. The language $\mathcal{L}_{S}^{*}$, used to describe structures of the form $(\gamma ; s, r)$ where $\gamma \in \mathrm{ON}, s: \gamma \rightarrow S$, and $r: \gamma \rightarrow S$ (partial), is the second order language generated through the following clauses:

This material is based upon work supported by the National Science Foundation under Grant No. DMS-0094174. 
1. $\alpha \in A, s(\alpha)=b, r(\alpha)=b, b \in \operatorname{cf}(s)$, and $b \in \operatorname{cf}(s\lceil\alpha)$ are atomic formulae of $\mathcal{L}_{S}^{*}$, where $\alpha$ is a first order variables, $A$ a second order variables, and $b$ an element of $S$.

2. If $\varphi$ and $\psi$ are formulae in $\mathcal{L}_{S}^{*}$ then so are $\neg \varphi$ and $(\varphi \wedge \psi)$.

3. If $\varphi$ is a formula in $\mathcal{L}_{S}^{*}$ then so is $(\exists A) \varphi$, where $A$ is a second order variable.

4. If $\varphi$ is a formula in $\mathcal{L}_{S}^{*}$ then so are $\left(\forall^{*} \alpha<\beta\right) \varphi$ and $\left(\forall^{*} \alpha\right) \varphi$, where $\alpha$ and $\beta$ are first order variables.

When a formula $\varphi$ in the language $\mathcal{L}_{S}^{*}$ is interpreted over the structure $(\gamma ; s, r)$, its first order variables range over elements of $\gamma$, and its second order variables range over subsets of $\gamma$. Truth value over $(\gamma ; s, r)$ is defined in the obvious way for formulae generated through conditions (1)-(3) of Definition 1.1. As for formulae generated through condition $(4):(\gamma ; s, r) \models\left(\forall^{*} \alpha<\beta\right) \varphi$ just in case that:

1. $\beta$ is a limit ordinal of cofinality at least $\omega_{1}$, and

2. there exists a club $C \subset \beta$ so that $(\gamma ; s, r) \models \varphi[\alpha]$ for all $\alpha \in C$.

$(\gamma ; s, r) \models\left(\forall^{*} \alpha\right) \varphi$ just in case that the same conditions hold, but with $\beta$ replaced by $\gamma$.

Claim 1.2. Let $\varphi$ be a sentence in $\mathcal{L}_{S}^{*}$. Then the truth value of $\varphi$ in a structure $(\gamma ; s, r)$ with $\gamma$ of cofinality $\omega$ (or a successor) depends only on $\operatorname{cf}(s)$.

Definition 1.3. Two structures $(\gamma ; s, r)$ and $\left(\gamma^{*} ; s^{*}, r^{*}\right)$ are similar, denoted $(\gamma ; s, r) \sim\left(\gamma^{*} ; s^{*}, r^{*}\right)$, if:

1. $\operatorname{cf}(s)=\operatorname{cf}\left(s^{*}\right)$.

2. There are clubs $C$ in $\gamma$ and $C^{*}$ in $\gamma^{*}$, and an order preserving bijection $f: C \rightarrow C^{*}$, so that $s^{*}(f(\xi))=s(\xi)$ and $r^{*}(f(\xi))=r(\xi)$ for all $\xi \in C$.

Claim 1.4. Let $\varphi$ be a sentence in $\mathcal{L}_{S}^{*}$. Let $(\gamma ; s, r)$ and $\left(\gamma^{*} ; s^{*}, r^{*}\right)$ be similar. Then $(\gamma ; s, r) \models \varphi$ iff $\left(\gamma^{*} ; s^{*}, r^{*}\right) \models \varphi$.

For proofs of Claims 1.2 and 1.4 see Neeman [4]. Using Claim 1.2 define $D \models \psi$, where $D \subset S$ and $\psi$ is a sentence of $\mathcal{L}_{S}^{*}$ to hold iff $(\gamma ; s, r) \models \psi$ for some (and hence all) structures $(\gamma ; s, r)$ with $\operatorname{cof}(\gamma)=\omega$ and $\operatorname{cf}(s)=D$.

Definition 1.5. Let $\Sigma$ be a finite non-empty set. A $\Sigma$-automaton is a tuple $\mathcal{A}=\langle S, P, T, \vec{\varphi}, \Psi, h, u\rangle$ where:

1. $S$ and $P$ are finite non-empty sets.

2. $T \subset S \times \Sigma \times S$.

3. $\vec{\varphi}=\left\langle\varphi_{1}, \ldots, \varphi_{k}\right\rangle$ is a finite tuple of sentences in $\mathcal{L}_{S}^{*}$.

4. $\Psi$ is a function from $2^{k}$ into $S$, where $k=\ln (\vec{\varphi})$.

5. $u$ is a function from $S$ into $\{U \mid U \subsetneq P\}$.

6. $h$ is a function from $S$ into $P$ with the property that $h(b) \in P-u(b)$ for each $b \in S$.

$\mathcal{A}$ is called deterministic if $T$ is a function from $S \times \Sigma$ into $S$, meaning that for each pair $\langle b, \sigma\rangle \in S \times \Sigma$ there is precisely one $b^{*} \in S$ so that $\left\langle b, \sigma, b^{*}\right\rangle \in T$.

We refer to $\Sigma$ as the alphabet, to $S$ as the set of states of $\mathcal{A}$, and to $P$ as the set of pebbles. $T$ is the successor transition table. $\vec{\varphi}$ and $\Psi$ determine limit transitions in a way that we explain below. $h$ and $u$ determine the placement and maintenance of pebbles. 
Definition 1.6. Let $\vec{\varphi}$ and $\Psi$ be as in conditions (3) and (4) above. Given a domain $(\gamma ; s, r)$ with $\gamma \in \mathrm{ON}, s: \gamma \rightarrow S$, and $r: \gamma \rightarrow S$, define $t_{(\gamma ; s, r)}^{\vec{\varphi}}: k \rightarrow 2$ by setting $t_{(\gamma ; s, r)}^{\vec{\varphi}}(i)=1$ if $(\gamma ; s, r) \models \varphi_{i}$ and $t_{(\gamma ; s, r)}^{\vec{\varphi}}(i)=0$ otherwise for each $i \leq k$. Define a function $\Psi \oplus \vec{\varphi}$, acting on domains $(\gamma ; s, r)$ as above, by setting $(\Psi \oplus \vec{\varphi})(\gamma ; s, r)=\Psi\left(t_{(\gamma ; s, r)}^{\vec{\varphi}}\right)$.

REMARK 1.7. For $D \subset S$ set $t_{D}^{\vec{\varphi}}(i)=1$ if $D \models \varphi_{i}$ and $t_{D}^{\vec{\varphi}}(i)=0$ otherwise. Set $(\Psi \oplus \vec{\varphi})(D)=\Psi\left(t_{D}^{\vec{\varphi}}\right)$. For $\gamma$ of cofinality $\omega$ then, $(\Psi \oplus \vec{\varphi})(\gamma ; s, r)=(\Psi \oplus \vec{\varphi})(\operatorname{cf}(s))$.

Definition 1.8. Let $\alpha$ be an ordinal and let $X: \alpha \rightarrow \Sigma$. A pair $\langle s, r\rangle$ where $s: \alpha+1 \rightarrow S$ and $r: \alpha \rightarrow S$ is called a run of $\mathcal{A}$ on $X$ just in case that it satisfies the following conditions:

(S) $\langle s(\xi), X(\xi), s(\xi+1)\rangle \in T$ for each $\xi<\alpha$.

(L) $s(\lambda)=(\Psi \oplus \vec{\varphi})(\lambda ; s\lceil\lambda, r \uparrow \lambda)$ for each limit $\lambda \leq \alpha$.

(R) If there exists some $\gamma>\xi$ so that $h(s(\xi)) \notin u(s(\gamma))$ then $r(\xi)=s(\gamma)$ for the least such $\gamma$, and otherwise $r(\xi)$ is undefined.

Condition (S) governs successor transitions, condition (L) governs limit transitions, and condition $(\mathrm{R})$ determines values for $r$.

The $\Sigma$-automaton $\mathcal{A}$ should be viewed as running over the input $X: \alpha \rightarrow \Sigma$ and producing a run $\langle s, r\rangle$ through a transfinite sequence of stages. In each stage $\beta$ the automaton determines $s(\beta)$ through either condition $(\mathrm{S})$ or condition $(\mathrm{L})$, depending on whether $\beta$ is a successor or a limit. In the case of successor $\xi+1$, the automaton determines the state $s(\xi+1)$ based on the previous state $s(\xi)$ and the input $X(\xi)$. The transition table $T$ dictates the possible choices, as $s(\xi+1)$ must be picked to that $\langle s(\xi), X(\xi), s(\xi+1)\rangle \in T$. In the case of a limit $\lambda$, the automaton determines $s(\lambda)$ based on a bounded fragment of the almostall theory of the run $(\lambda ; s \uparrow \lambda, r\lceil\lambda)$ produced so far. The fragment consulted is the restriction of the theory to the sentences in $\vec{\varphi}$. The function $\Psi$ tells the automaton how to set the state $s(\lambda)$ based on this fragment.

Having determined $s(\beta)$, the automaton places the pebble $p=h(s(\beta))$ on the ordinal $\beta$. The pebble $p$ remains placed on $\beta$ until a later stage $\beta^{*}$ is reached with $p \notin u\left(s\left(\beta^{*}\right)\right)$. At the first such stage $\beta^{*}$ the automaton removes the pebble from $\beta$, and sets $r(\beta)=s\left(\beta^{*}\right)$. This is expressed precisely in condition $(\mathrm{R})$. $r(\beta)$ remains undefined until the pebble placed on $\beta$ is removed, and may indeed remain undefined throughout, if the pebble is not removed at all during the run. The use of pebbles therefore introduces a delay into part of the construction of a run. This delay is essential in the proof of Theorems 1.10 and 1.11 below.

Notice that the value of $r\left\lceil\lambda\right.$ known by stage $\lambda$ - call it $(r \uparrow \lambda)^{\text {local }}$ - is not the same as the final value $r\lceil\lambda$ known by the end of the run, after stage $\alpha$, as there may be ordinals $\xi<\lambda$ so that the pebble $h(s(\xi))$ placed on $\xi$ is removed at a stage $\gamma \geq \lambda$. But there may only be finitely many such ordinals, since the number of pebbles is finite and since no pebble is ever located on two ordinals at the same stage (to see this use the restriction $h(b) \notin u(b)$ in condition (6) of Definition 1.5). Thus $\left(r\lceil\lambda)^{\text {local }}\right.$ and $r\lceil\lambda$ may only differ on a finite set.

When reaching a limit stage $\lambda$ the automaton looks at the value of $r\lceil\lambda$ known by stage $\lambda$, setting $s(\lambda)$ equal to $(\Psi \oplus \vec{\varphi})\left(\lambda ; s \uparrow \lambda,(r \uparrow \lambda)^{\text {local }}\right)$. This assignment 
satisfies condition (L) in Definition 1.8 since, by Claim 1.4 and the fact that $\left(r\lceil\lambda)^{\text {local }}\right.$ and $r\left\lceil\lambda\right.$ differ only on a finite set, the structures $\left(\lambda ; s\left\lceil\lambda,\left(r\lceil\lambda)^{\text {local }}\right)\right.\right.$ and $(\lambda ; s \uparrow \lambda, r \uparrow \lambda)$ satisfy precisely the same sentences.

REMARK 1.9. Coding runs and inputs by sets of ordinals, one can express the existence of a run starting at a given state $b$ and ending at a given state $b^{*}$ in the monadic second order language. To be precise, for $X: \theta \rightarrow \Sigma$ and $\sigma \in \Sigma$ set $A_{X, \sigma}=\{\xi \mid X(\xi)=\sigma\}$. Let $\sigma_{1}, \ldots, \sigma_{l}$ enumerate $\Sigma$. Then for every $b, b^{*} \in S$ there is a formula $\varphi_{b, b^{*}}$ so that (for all $\theta$ and all $X: \theta \rightarrow \Sigma$ ) $(\theta ;<) \models \varphi_{b, b^{*}}\left[A_{X, \sigma_{1}}, \ldots, A_{X, \sigma_{l}}\right]$ iff there is a run $\langle s, r\rangle$ of $\mathcal{A}$ on $X$ with $s(0)=b$ and $s(\theta)=b^{*}$.

An accepting condition for an automaton $\mathcal{A}$ is a pair $\langle I, F\rangle$ where $I \in S$ and $F \subset S .\langle\mathcal{A}, I, F\rangle$ is said to accept $X: \alpha \rightarrow \Sigma$ just in case that there exists a run $\langle s, r\rangle$ of $\mathcal{A}$ on $X$ so that $s(0)=I$ and $s(\alpha) \in F$. Notice that if $\mathcal{A}$ is deterministic then it has exactly one run $\langle s, r\rangle$ on $X$ with $s(0)=I$, so that $\langle\mathcal{A}, I, F\rangle$ accepts $X$ iff $s(\ln (X)) \in F$ for $s$ taken from this unique run.

Call $\langle\mathcal{A}, I, F\rangle$ and $\left\langle\mathcal{A}^{*}, I^{*}, F^{*}\right\rangle$ equivalent if for every ordinal $\alpha$ and every $X: \alpha \rightarrow \Sigma,\langle\mathcal{A}, I, F\rangle$ accepts $X$ iff $\left\langle\mathcal{A}^{*}, I^{*}, F^{*}\right\rangle$ accepts $X$.

TheORem 1.10 (Neeman [4]). For any automaton $\mathcal{A}$ and accepting condition $\langle I, F\rangle$, there is a deterministic automaton $\mathcal{A}^{*}$ with accepting condition $\left\langle I^{*}, F^{*}\right\rangle$ so that $\langle\mathcal{A}, I, F\rangle$ and $\left\langle\mathcal{A}^{*}, I^{*}, F^{*}\right\rangle$ are equivalent.

Theorem 1.10 extends the work of Büchi [1] and Büchi-Zaiontz [2] to automata acting on inputs of lengths $\omega_{2}$ and greater. The specific details of the definition of automata above are of course important to the proof of the theorem.

For $a \in \mathrm{ON}$ define $\chi_{\mathrm{f}}(a): \mathrm{ON} \rightarrow 2$ through the condition $\chi_{\mathrm{f}}(a)(\gamma)=1$ if $\gamma=a$ and $\chi_{\mathrm{f}}(a)(\gamma)=0$ otherwise. For $a \subset \mathrm{ON}$ define $\chi_{\mathrm{s}}(a): \mathrm{ON} \rightarrow 2$ through the condition $\chi_{\mathbf{s}}(a)(\gamma)=1$ if $\gamma \in a$ and $\chi_{\mathrm{s}}(a)(\gamma)=0$ otherwise. ( $\mathrm{f}$ and $\mathrm{s}$ here stand for "first order" and "second order.") Given a monadic second order formula $\varphi$ with free variables $x_{1}, \ldots, x_{k}$ let $\operatorname{sig}(\varphi): k \rightarrow\{\mathrm{s}, \mathrm{f}\}$ be the function defined by the condition $\operatorname{sig}(\varphi)(i)=\mathrm{s}$ if $x_{i}$ is a second order variable, and $\operatorname{sig}(\varphi)(i)=\mathrm{f}$ if $x_{i}$ is a first order variable. A sequence $\left\langle a_{1}, \ldots, a_{k}\right\rangle$ fits the signature of $\varphi$ if $a_{i}$ is an ordinal for $i$ such that $\operatorname{sig}(\varphi)(i)=\mathrm{f}$, and a set or class of ordinals for $i$ such that $\operatorname{sig}(\varphi)(i)=\mathrm{s}$. Given a sequence $\left\langle a_{1}, \ldots, a_{k}\right\rangle$ which fits the signature of $\varphi$ define $\chi\left(a_{1}, \ldots, a_{k}\right): \mathrm{ON} \rightarrow 2^{k}$ through the condition $\chi\left(a_{1}, \ldots, a_{k}\right)(\gamma)=$ $\left\langle\chi_{\operatorname{sig}(\varphi)(1)}\left(a_{1}\right)(\gamma), \ldots, \chi_{\operatorname{sig}(\varphi)(k)}\left(a_{k}\right)(\gamma)\right\rangle$. This is the characteristic function of $\left\langle a_{1}, \ldots, a_{k}\right\rangle$.

The following theorem, essentially a converse to Remark 1.9, completes the connection between monadic second order formulae over the ordinals and finite state automata. The theorem is proved by induction on the complexity of $\varphi$. The case of existential quantification is easy when working with non-deterministic automata, and the case of negation is easy when working with deterministic automata. Thus the bulk of the work in reaching Theorem 1.11 is securing Theorem 1.10, namely the equivalence between deterministic and non-deterministic automata.

ThEOREM 1.11 (Neeman [4]). Let $\varphi$ be a monadic second order formula in the language of order, with $k$ free variables say. Then there is a deterministic finite 
state automaton $\mathcal{A}$, with accepting condition $\langle I, F\rangle$, so that: for every ordinal $\theta$ (also for $\theta=\mathrm{ON}$ ), and for every sequence $a_{1}, \ldots, a_{k}$ which fits the signature of $\varphi,(\theta ;<) \models \varphi\left[a_{1}, \ldots, a_{k}\right]$ iff $\langle\mathcal{A}, I, F\rangle$ accepts $\chi\left(a_{1}, \ldots, a_{k}\right)\lceil\theta$.

Call an ordinal $\theta$ definable if there is a monadic formula $\varphi(v)$ so that $(\mathrm{ON} ;<) \models$ $\varphi[\alpha]$ iff $\alpha=\theta$, and definable with parameters $x_{1}, \ldots, x_{k}$ if there is a formula $\varphi\left(v_{1}, \ldots, v_{k}, v\right)$ so that $(\mathrm{ON} ;<) \models \varphi\left[x_{1}, \ldots, x_{k}, \alpha\right]$ iff $\alpha=\theta$.

An ordinal $\theta$ can be pinpointed iff there is a sentence $\psi$ so that $(\theta ;<) \models \psi$ and for all $\alpha<\theta,(\alpha ;<) \forall \forall \psi . \psi$ is said to pinpoint $\theta$.

It is clear that ordinals which can be pinpointed are definable. Using Theorem 1.11 one can prove the converse:

Lemma 1.12 (Neeman [4]). If $\theta$ is definable then it can be pinpointed.

For $\alpha \in \mathrm{ON}$ let $0^{\alpha}$ denote the input $X: \alpha \rightarrow\{0\}$ defined by $X(\xi)=0$ for all $\xi$. If $\theta$ can be pinpointed then by Theorem 1.11 there is a deterministic automaton $\mathcal{A}$ and accepting condition $\langle I, F\rangle$ so that $\langle\mathcal{A}, I, F\rangle$ accepts $0^{\theta}$ and does not accept $0^{\alpha}$ for any $\alpha<\theta$. This makes the following claim useful in the study of monadic definability of ordinals:

Claim 1.13 (Neeman [4]). Let $\mathcal{A}$ be a deterministic automaton and let $\langle s, r\rangle$ be a run of $\mathcal{A}$ on $0^{\theta}$. Let $D=\operatorname{cf}(s \mid \theta)$. Let $\delta<\theta$ be least so that $\{s(\xi) \mid \delta \leq \xi<$ $\theta\}=D$. Let $\gamma$ be least so that $\{s(\xi) \mid \delta \leq \xi<\delta+\gamma\}=D$ and $s(\delta+\gamma)=s(\delta)$. Let $C=\{\alpha \in(\delta, \theta] \mid \alpha$ is closed under addition of $\gamma\}$. Then $\operatorname{cf}(s\lceil\alpha)=D$ for every $\alpha \in C$, and, for $\alpha, \beta \in C$, if $\operatorname{cof}(\alpha)=\operatorname{cof}(\beta)$ then $s(\alpha)=s(\beta)$.

Notice that, in the notation of the last claim, $\theta$ is closed under addition of $\gamma$ (else $\operatorname{cf}(s\lceil\theta)$ would not be equal to $D$ ). Thus it follows from the claim that either $\theta=\delta+\gamma \cdot \operatorname{cof}(\theta)$, or else there is $\alpha<\theta$, namely $\alpha=\delta+\gamma \cdot \operatorname{cof}(\theta)$, so that $s(\alpha)=s(\theta)$. If $\mathcal{A}$ and $\langle I, F\rangle$ are obtained from Theorem 1.11 using a formula which pinpoints $\theta$, and $\langle s, r\rangle$ is an accepting run of $\langle\mathcal{A}, I, F\rangle$, then the latter is impossible. Thus:

Claim 1.14 (Neeman [4]). Suppose that $\theta$ is definable. Then there is a deterministic automaton $\mathcal{A}$ and a run $\langle s, r\rangle$ of $\mathcal{A}$ on $0^{\theta}$ so that $\theta=\delta+\gamma \cdot \operatorname{cof}(\theta)$ where $\delta$ and $\gamma$ are defined from $s$ as in Claim 1.13.

In particular, no singular cardinal can be definable. Neeman [4] concludes with this result.

§2. Definability, forward. In this section we make the simple observation that the set of definable ordinals is closed under ordinal addition and multiplication. This is true even though neither addition nor multiplication is a definable operation, and $\alpha+\beta$ need not in general be definable from $\alpha$ and $\beta$, as we shall see in Section 4. (Recall that definability here is in the monadic language.)

Given a monadic formula $\varphi\left(v_{1}, \ldots, v_{k}\right)$, let $\varphi^{\mathrm{rel}}\left(v_{1}, \ldots, v_{k}, A\right)$ be the formula obtained from $\varphi$ by replacing all first order quantifiers $\left(\exists v_{i}\right)$ and $\left(\forall v_{i}\right)$ in $\varphi$ with (the formal equivalent of) $\left(\exists v_{i} \in A\right)$ and $\left(\forall v_{i} \in A\right)$.

Claim 2.1. For a sentence $\psi,(\mathrm{ON} ;<) \models \psi^{\mathrm{rel}}[A]$ iff $(\operatorname{ot}(A) ;<) \models \psi$, where $\operatorname{ot}(A)$ is the order type of $A$. 
Proof. Fix $A$. Let $f: \operatorname{ot}(A) \rightarrow A$ be the unique order preserving bijection. Given a formula $\varphi\left(v_{1}, \ldots, v_{k}\right)$, call $x_{1}, \ldots, x_{k}$ and $x_{1}^{*}, \ldots, x_{k}^{*}$ similar if $x_{i} \in$ ot $(A)$ and $x_{i}^{*}=f\left(x_{i}\right)$ for $i$ such that $v_{i}$ is first order, and $x_{i} \subset \operatorname{ot}(A)$ and $x_{i}^{*} \cap A=$ $f^{\prime \prime}\left(x_{i}\right)$ for $i$ such that $v_{i}$ is second order. Then for every formula $\varphi\left(v_{1}, \ldots, v_{k}\right)$, $(\operatorname{ot}(A) ;<) \models \varphi\left[x_{1}, \ldots, x_{k}\right]$ iff $(\mathrm{ON} ;<) \models \varphi^{\mathrm{rel}}\left[x_{1}^{*}, \ldots, x_{k}^{*}, A\right]$ whenever $x_{1}, \ldots, x_{k}$ and $x_{1}^{*}, \ldots, x_{k}^{*}$ are similar. This statement is easily proved by induction on the complexity of $\varphi$, and the case of $k=0$ yields the claim.

Claim 2.2. Let $\alpha$ and $\beta$ be ordinals. Suppose that $\alpha$ is definable with parameters $x_{1}, \ldots, x_{k}$ and that $\beta$ is definable. Then $\alpha+\beta$ is definable with parameters $x_{1}, \ldots, x_{k}$.

Proof. Let $\psi_{\beta}$ pinpoint $\beta$. Let $\psi_{\alpha}\left(v_{1}, \ldots, v_{k}, v\right)$ witness that $\alpha$ is definable with parameters $x_{1}, \ldots, x_{k}$. Let $\varphi\left(v_{1}, \ldots, v_{k}, y\right)$ be the formula

$$
\begin{aligned}
(\exists \eta)(\exists A)\left(\psi_{\alpha}\left(v_{1}, \ldots, v_{k}, \eta\right) \wedge(\xi \in A \Longleftrightarrow\right. & \eta \leq \xi<y) \wedge \\
& \left.\psi_{\beta}^{\text {rel }}(A) \wedge(\forall u<y) \neg \psi_{\beta}^{\text {rel }}(A \cap u)\right)
\end{aligned}
$$

Then $(\mathrm{ON} ;<) \models \varphi\left[x_{1}, \ldots, x_{k}, \theta\right]$ iff $\theta=\alpha+\beta$.

Claim 2.3. Let $\alpha$ and $\beta$ be definable ordinals. Then $\alpha \cdot \beta$ is definable.

Proof. Fix $\psi_{\alpha}$ and $\psi_{\beta}$ which pinpoint $\alpha$ and $\beta$ respectively. Then $\theta=\alpha \cdot \beta$ iff there exists $A$ so that:

- $A$ is a closed unbounded subset of $\theta+1$ and 0 and $\theta$ are both in $A$.

- $\psi_{\beta}^{\mathrm{rel}}[A \cap \theta]$ holds, and for every $u<\theta, \psi_{\beta}^{\mathrm{rel}}[A \cap u]$ fails. So ot $(A \cap \theta)=\beta$.

- $\psi_{\alpha}^{\text {rel }}[C]$ holds whenever $C=\left[\zeta, \zeta^{*}\right)$ with $\zeta \in A$ and $\zeta^{*}$ equal to the first element of $A$ above $\zeta$, and $\psi_{\alpha}^{\mathrm{rel}}[C \cap u]$ fails for all $u<\zeta^{*}$. So ot $(C)=\alpha$.

These conditions can be phrased in the monadic language, providing a formula that defines $\alpha \cdot \beta$.

An ordinal is a multiple of $\gamma$ if it has the form $\gamma \cdot \nu$ for some ordinal $\nu$.

Claim 2.4. Suppose that $\gamma$ is definable. Then there is a formula $\varphi(v)$ so that $(\mathrm{ON} ;<) \models \varphi[\theta]$ iff $\theta$ is a multiple of $\gamma$.

Proof. Similar to the proof of the previous claim.

Given ordinals $\gamma$ and $\alpha$ define $\operatorname{trunc}_{\gamma}(\alpha)$ to be the largest multiple of $\gamma$ which is $\leq \alpha$. To give just two examples, $\operatorname{trunc}_{1}(\alpha)=\alpha$ and $\operatorname{trunc}_{0}(\alpha)=0$.

Claim 2.5. Suppose that $\gamma$ is definable. Then $\operatorname{trunc}_{\gamma}(\alpha)$ is definable with parameter $\alpha$.

ProOF. Immediate from the last claim.

Claims 2.2 and 2.5 show that given a class $P \subset \mathrm{ON}$, every ordinal of the form trunc ${ }_{\gamma}(\alpha)+\beta$, where $\alpha \in P$ and $\gamma, \beta$ are definable with no parameters, is definable with parameters from $P$. We shall see later that in fact these are all the ordinals definable with parameters from $P$. 
§3. Definability, backward. Let $\theta$ be a definable limit ordinal. Let $\psi$ pinpoint $\theta$. Using Theorem 1.11 fix a deterministic automaton $\mathcal{A}$ and an accepting condition $\langle I, F\rangle$ so that $\langle\mathcal{A}, I, F\rangle$ accepts $0^{\alpha}$ iff $(\alpha ;<) \models \psi$. Let $\langle s, r\rangle$ be the unique run of $\mathcal{A}$ on $0^{\theta}$ with $s(0)=I$. As in Claim 1.13 set:

(i) $D=\operatorname{cf}(s\lceil\theta)$.

(ii) $\delta$ is least so that $\{s(\xi) \mid \delta \leq \xi<\theta\}=D$.

(iii) $\gamma$ is least so that $\{s(\xi) \mid \delta \leq \xi<\delta+\gamma\}=D$ and $s(\delta+\gamma)=s(\delta)$.

Let $b^{*}=s(\theta)$ and notice that $s(\alpha) \neq b^{*}$ for $\alpha<\theta$ since $\langle\mathcal{A}, I, F\rangle$ does not accept $0^{\alpha}$. Let $C=\{\delta+\gamma \cdot \omega \cdot \xi \mid \xi \geq 1 \wedge \delta+\gamma \cdot \omega \cdot \xi \leq \theta\}$. $\theta$ belongs to $C$ and by Claim 1.13 there is no $\alpha<\theta$ in $C$ with $\operatorname{cof}(\alpha)=\operatorname{cof}(\theta)$. Hence $\theta=\delta+\gamma \cdot \operatorname{cof}(\theta)$.

ClAIM 3.1. $\delta$ and $\gamma$ are definable.

Proof. By Remark 1.9, and using the fact that $\mathcal{A}$ is deterministic, there is for each $b \in S$ a monadic formula $\varphi_{b}(v)$ so that $(\mathrm{ON} ;<) \models \varphi_{b}[\xi]$ iff $s(\xi)=b$. Conditions (ii) and (iii) can thus be phrased in the monadic language, providing a definition of $\gamma$, and a definition of $\delta$ from $\theta$. Since $\theta$ is definable, both $\delta$ and $\gamma$ are definable.

Lemma 3.2. $\operatorname{cof}(\theta)$ is definable.

Proof. We may assume that $\operatorname{cof}(\theta)>\omega$, since otherwise $\operatorname{cof}(\theta)$ is clearly definable. Let $X$ denote $0^{\theta}$, so that $\langle s, r\rangle$ is a run of $\mathcal{A}$ on $X$. Note to begin with that for every $\alpha, \beta \in(\delta, \theta)$, if $s(\alpha)=s(\beta)$ then $r(\alpha)=r(\beta)$. In fact, if $s(\alpha)=s(\beta)$ then $s(\alpha+\xi)=s(\beta+\xi)$ for all $\xi$ so that $\alpha+\xi, \beta+\xi<\theta$, since $\mathcal{A}$ is deterministic and $X(\alpha+\xi)=X(\beta+\xi)$. From this and the fact that $\{s(\zeta) \mid \alpha<\zeta<\theta\}=\{s(\zeta) \mid \beta<\zeta<\theta\}$ it follows that $r(\alpha)=r(\beta)$.

Thus there is a function $R: S \rightarrow S$ so that, for $\alpha \in(\delta, \theta), r(\alpha)=R(s(\alpha))$.

Let $\langle S, P, T, \vec{\varphi}, \Psi, h, u\rangle$ constitute the automaton $\mathcal{A}$. For each $i<\ln (\vec{\varphi})$ let $\varphi_{i}^{*}$ be obtained from $\varphi_{i}$ by replacing each occurrence of $r(\xi)=b$ with $\bigvee_{\bar{b} \in R^{-1}(b)} s(\xi)=\bar{b}$. Then:

(iv) For each limit $\lambda \in(\delta, \theta],\left(\Psi \oplus \vec{\varphi}^{*}\right)(\lambda ; s\lceil\lambda, r\lceil\lambda)=(\Psi \oplus \vec{\varphi})(\lambda ; s\lceil\lambda, r\lceil\lambda)$.

(v) The sentences in $\vec{\varphi}^{*}$ make no mention of $r$.

Let $b_{1}, \ldots, b_{j}$ enumerate the states in $D$. Set $T^{*}=\left\{\left\langle b_{i}, 0, b_{i+1}\right\rangle \mid i<j\right\} \cup$ $\left\{\left\langle b_{j}, 0, b_{1}\right\rangle\right\}$. Let $\mathcal{A}^{*}$ be the automaton $\left\langle S, P, T^{*}, \vec{\varphi}^{*}, \Psi, h, u\right\rangle$. Let $\tau=\operatorname{cof}(\theta)$ and let $\left\langle s^{*}, r^{*}\right\rangle$ be the unique run of $\mathcal{A}^{*}$ on $0^{\tau}$ with $s^{*}(0)=s(\delta)$.

Define $r^{\prime}: \theta \rightarrow S$ by setting $r^{\prime}(\delta+\gamma \cdot \omega \cdot \xi)=r^{*}(\omega \cdot \xi)$ and leaving $r^{\prime}(\zeta)$ undefined on $\zeta$ not covered by this clause.

Claim 3.3. For every $\xi \leq \tau$ :

1. $s(\delta+\gamma \cdot \omega \cdot \xi)=s^{*}(\omega \cdot \xi)$.

2. Let $\zeta=\delta+\gamma \cdot \omega \cdot \xi$. Then for $\xi \geq 1,\left(\zeta ; s \uparrow \zeta, r^{\prime} \uparrow \zeta\right)$ and $\left(\omega \cdot \xi ; s^{*} \uparrow \omega \cdot \xi, r^{*} \uparrow \omega \cdot \xi\right)$ are similar.

Proof. The proof is by induction on $\xi$. Condition (1) for $\xi=0$ follows from the definition, as $s^{*}(0)$ was set equal to $s(\delta)$. Condition (1) for $\xi \geq 1$ follows from condition (2) for $\xi$ together with conditions (iv) and (v) above and Claim 1.4. Condition (2) for $\xi$ of cofinality $\leq \omega$ follows simply from the fact that $\operatorname{cf}(s \uparrow \zeta)=\operatorname{cf}\left(s^{*} \uparrow \omega \cdot \xi\right)$. (Both are equal to $D$, by Claim 1.13 in the case of 
$\operatorname{cf}\left(s\lceil\zeta)\right.$ and by the definition of $T^{*}$ in the case of $\operatorname{cf}\left(s^{*} \uparrow \omega \cdot \xi\right)$.) For $\xi$ of cofinality $>\omega$ condition (2) follows from the fact that $\operatorname{cf}\left(s\lceil\zeta)=\operatorname{cf}\left(s^{*} \uparrow \omega \cdot \xi\right)\right.$, and from condition (1) below $\xi$, which gives clubs below $\zeta$ and $\omega \cdot \xi$ on which $s \uparrow \zeta$ and $s^{*} \uparrow \omega \cdot \xi$ are equal.

Since $s(\theta)=b^{*}$ and $s(\alpha) \neq b^{*}$ for $\alpha<\theta$, it follows from the last claim that $\tau=\operatorname{cof}(\theta)$ is least so that $s^{*}(\tau)=b^{*}$. Using Remark 1.9 this can be turned into

a definition of $\tau$ in the monadic second order language. $\quad-$ (Lemma 3.2)

Theorem 3.4. Let $\theta$ be an ordinal. Then $\theta$ is definable iff $\theta=0$ or the following conditions hold:

1. $\operatorname{cof}(\theta)$ is definable.

2. There are definable ordinals $\delta, \gamma<\theta$ so that $\theta=\delta+\gamma \cdot \operatorname{cof}(\theta)$.

Proof. The right-to-left direction is immediate from the results in Section 2. The left-to-right direction is clear for 0 and for successor $\theta$, and follows from Claim 3.1 and Lemma 3.2 for limit $\theta$.

Theorem 3.5. An ordinal is definable iff it can be obtained from definable regular cardinals using ordinal addition and multiplication.

Proof. Again the right-to-left direction is immediate from the results in Section 2. The left-to-right direction is proved by induction using Theorem 3.4.

§4. Parameters. Let $a_{1}<\cdots<a_{l}$ be ordinals, and let $\theta \notin\left\{a_{1}, \ldots, a_{l}\right\}$ be a limit ordinal definable with parameters $a_{1}, \ldots, a_{l}$. Let $\varphi$ be a monadic formula such that $(\mathrm{ON} ;<) \models \varphi\left[a_{1}, \ldots, a_{l}, \alpha\right]$ iff $\alpha=\theta$.

Adding a bogus parameter if needed we may assume that there is $k<l$ so that $a_{k}<\theta<a_{k+1}$. For $\alpha \in\left(a_{k}, a_{k+1}\right)$ define $X_{\alpha}$ : ON $\rightarrow 2$ through the conditions $X\left(a_{i}\right)=1$ for each $i, X(\alpha)=1$, and $X(\xi)=0$ for $\xi \notin\left\{\alpha, a_{1}, \ldots, a_{l}\right\}$.

Using Theorem 1.11 find a deterministic automaton $\mathcal{A}$ and an accepting condition $\langle I, F\rangle$ so that, for each $\alpha \in\left(a_{k}, a_{k+1}\right),\langle\mathcal{A}, I, F\rangle$ accepts $X_{\alpha}$ iff $(\mathrm{ON} ;<) \models$ $\varphi\left[a_{1}, \ldots, a_{l}, \alpha\right]$.

Let $\langle s, r\rangle$ be the unique run of $\mathcal{A}$ on $X_{\theta}$ with $s(0)=I$. Set $D=\operatorname{cf}(s \uparrow \theta)$, $\delta>a_{k}$ least so that $\{s(\xi) \mid \delta \leq \xi<\theta\}=D$, and $\gamma$ least so that $\{s(\xi) \mid \delta \leq \xi<$ $\delta+\gamma\}=D$ and $s(\delta+\gamma)=s(\bar{\delta})$.

Let $b^{*}=s(\theta)$. Let $C=\{\delta+\gamma \cdot \omega \cdot \xi \mid \xi \geq 1\}$. Note that $\theta$ is closed under addition of $\gamma$, so $\theta \in C$. Let $\tau$ be least so that $s(\delta+\gamma \cdot \omega \cdot \tau)=b^{*}$. By Claim 1.13, $s(\alpha)=s(\beta)$ for $\alpha, \beta \in C$ of the same cofinality, so $\tau$ is a regular cardinal, and $s(\delta+\gamma \cdot \xi)=b^{*}$ for all $\xi$ of cofinality $\tau$.

Claim 4.1. $\gamma$ is definable with no parameters, $\tau$ is definable with no parameters, and $\delta$ is definable with parameters $a_{1}, \ldots, a_{l}$.

Proof. Similar to the proofs of Claim 3.1 and Lemma 3.2.

Claim 4.2. Suppose that $\theta$ is not the first ordinal of cofinality $\tau$ in $C$. Then $a_{k+1}<\theta+\gamma \cdot \tau \cdot \omega$.

Proof. Suppose otherwise. Let $\alpha=\theta+\gamma \cdot \tau$. We show that $\langle\mathcal{A}, I, F\rangle$ accepts $X_{\alpha}$. Since $\alpha<a_{k+1}$ this implies that $(\mathrm{ON} ;<) \models \varphi\left[a_{1}, \ldots, a_{l}, \alpha\right]$, contradicting the fact that $\varphi$ defines $\theta$ from $a_{1}, \ldots, a_{l}$. 
Let $\left\langle s^{*}, r^{*}\right\rangle$ be the unique run of $\mathcal{A}$ on $X_{\alpha}$ with $s^{*}(0)=I$. Since $X_{\alpha}\left\lceil\theta=X_{\theta}\lceil\theta\right.$, $s$ and $s^{*}$ are the same up to an including $\theta$. Thus $s^{*}(\theta)=b^{*}$.

For $\zeta$ in the interval $[\theta, \alpha)$, that is the interval $[\theta, \theta+\gamma \cdot \tau), X_{\alpha}(\zeta)$ is equal to 0 . By assumption $\theta$ is not the least ordinal of cofinality $\tau$ in $C$, hence $\theta \geq \delta+\gamma \cdot \tau \cdot 2$, so that $X_{\theta}(\zeta)=0$ for $\zeta$ in the interval $[\delta+\gamma \cdot \tau, \delta+\gamma \cdot \tau \cdot 2)$.

Thus $X_{\alpha}(\theta+\xi)=X_{\theta}(\delta+\gamma \cdot \tau+\xi)$ for all $\xi<\gamma \cdot \tau$. It follows from this, the fact that $s^{*}(\theta)=s(\delta+\gamma \cdot \tau)$ (both are equal to $b^{*}$ ), and the determinism of $\mathcal{A}$, that $s^{*}(\theta+\xi)=s(\delta+\gamma \cdot \tau+\xi)$ for each $\xi \leq \gamma \cdot \tau$. In particular then $s^{*}(\theta+\gamma \cdot \tau)$ is equal to $s(\delta+\gamma \cdot \tau \cdot 2)$, and the latter is equal to $b^{*}$ since $\delta+\gamma \cdot \tau \cdot 2$ is in $C$ and of cofinality $\tau$.

We established so far that $s^{*}(\alpha)=s(\theta)$ (both are equal to $b^{*}$ ). Note that $X_{\alpha}(\alpha+\xi)=X_{\theta}(\theta+\xi)$ for every $\xi$ : for $\xi=0$ both are equal to 1 , for $\xi \in(0, \gamma \cdot \tau \cdot \omega)$ both are equal to 0 as $a_{l+1} \geq \theta+\gamma \cdot \tau \cdot \omega$ by assumption, and for $\xi \geq \gamma \cdot \tau \cdot \omega, \alpha+\xi$ is equal to $\theta+\xi$. From the fact that $X_{\alpha}(\alpha+\xi)=X_{\theta}(\theta+\xi)$ for all $\xi$, the fact that $s^{*}(\alpha)=s(\theta)$, and the determinism of $\mathcal{A}$ it follows that $s^{*}(\alpha+\xi)=s(\theta+\xi)$ for all $\xi$.

$s^{*}$ and $s$ are thus the same on a tail-end of ON. Since $\langle s, r\rangle$ is an accepting run of $\langle\mathcal{A}, I, F\rangle$, it follows that so is $\left\langle s^{*}, r^{*}\right\rangle$. $\langle\mathcal{A}, I, F\rangle$ therefore accepts $X_{\alpha}$, meaning that $(\mathrm{ON} ;<) \models \varphi\left[a_{1}, \ldots, a_{l}, \alpha\right]$, contradicting the fact that $\varphi$ defines $\theta$.

Claim 4.3. Either there is $n<\omega$ so that $\theta=\delta+\gamma \cdot \tau \cdot n$, or else there is $n<\omega$ so that $\theta=\operatorname{trunc}_{\gamma \cdot \tau \cdot \omega}\left(a_{k+1}\right)+\gamma \cdot \tau \cdot n$.

Proof. The choice of $\tau$ above is such that $\theta$ has the form $\delta+\gamma \cdot \xi$ for some $\xi$ of cofinality $\geq \tau$. Suppose that $\theta$ is not equal to $\delta+\gamma \cdot \tau \cdot n$ for any $n$. Then $\theta$ must have the form $\delta+\gamma \cdot \tau \cdot \xi$ for $\xi \geq \omega$, and since $\delta+\gamma \cdot \tau \cdot \omega$ is a multiple of $\gamma \cdot \tau$ we may drop $\delta$, concluding that $\theta$ has the form $\gamma \cdot \tau \cdot \xi$ for some $\xi$.

Recall that trunc $\gamma \cdot \tau \cdot \omega\left(a_{k+1}\right)$ is the largest multiple of $\gamma \cdot \tau \cdot \omega$ which is $\leq a_{k+1}$. Since $\theta$ is not equal to $\delta+\gamma \cdot \tau$, it follows from the previous claim that $\theta \geq$ trunc $_{\gamma \cdot \tau \cdot \omega}\left(a_{k+1}\right) . \theta<a_{k+1}$ and $a_{k+1}$ of course is smaller than trunc ${ }_{\gamma \cdot \tau \cdot \omega}\left(a_{k+1}\right)+$ $\gamma \cdot \tau \cdot \omega$. Thus $\theta$ belongs to the interval [trunc $\left.\gamma \cdot \tau \cdot \omega\left(a_{k+1}\right), \operatorname{trunc}_{\gamma \cdot \tau \cdot \omega}\left(a_{k+1}\right)+\gamma \cdot \tau \cdot \omega\right)$. From this and the fact that $\theta$ is a multiple of $\gamma \cdot \tau$ it follows that $\theta$ has the form trunc $_{\gamma \cdot \tau \cdot \omega}\left(a_{k+1}\right)+\gamma \cdot \tau \cdot n$ for some $n<\omega$.

Corollary 4.4. Let $\theta$ be definable with parameters $a_{1}, \ldots, a_{l}$. Then at least one of the following conditions holds:

1. There is $\delta<\theta$ definable with parameters $a_{1}, \ldots, a_{l}$, and an ordinal $\beta$ definable with no parameters, so that $\theta=\delta+\beta$.

2. There is $i \leq l$ and ordinals $\alpha, \beta$ definable with no parameters, so that $\theta=$ $\operatorname{trunc}_{\alpha}\left(a_{i}\right)+\beta$.

Proof. If $\theta$ is a successor ordinal then condition (1) holds with $\delta=\theta-1$. If $\theta=0$ then condition (2) holds with $\alpha=\beta=0$. If $\theta \in\left\{a_{1}, \ldots, a_{l}\right\}$ then condition (2) holds with $\alpha=1$ and $\beta=0$. Finally, for $\theta$ a limit ordinal not in $\left\{a_{1}, \ldots, a_{l}\right\}$, the corollary follows from the previous claim using the fact that $\gamma$ and $\tau$ (an hence also $\gamma \cdot \tau, \gamma \cdot \tau \cdot n$ for each $n<\omega$, and $\gamma \cdot \tau \cdot \omega$ ) are definable with no parameters. 
TheOREM 4.5. Let $P \subset \mathrm{ON}$ be a non-empty class. Then $\theta$ is definable with parameters from $P$ iff it belongs to the class $\left\{\operatorname{trunc}_{\gamma}(\alpha)+\beta \mid \alpha \in P\right.$ and $\gamma, \beta$ are definable with no parameters\}.

Proof. The right-to-left direction follows from the results in Section 2. The left-to-right follows from Corollary 4.4 by induction on $\theta$.

Theorem 4.5 shows that very little can be gained from parameters in the case of monadic definability over $(\mathrm{ON} ;<)$. It has the following immediate consequences:

Claim 4.6. There are ordinals $\alpha, \beta$ so that $\alpha+\beta$ is not definable with parameters $\alpha, \beta$. There are ordinals $\alpha, \beta$ so that $\alpha \cdot \beta$ is not definable with parameters $\alpha, \beta$. There is an ordinal $\alpha$ so that $\operatorname{cof}(\alpha)$ is not definable with parameter $\alpha$.

PRoOF. The ordinal $\omega^{\omega}$ is (by Theorem 3.5) a multiple of all the definable countable ordinals, and not itself definable. It follows from Theorem 4.5 that $\omega^{\omega}+\omega^{\omega}$ is not definable with parameter $\omega^{\omega}$ (and, equivalently, $\omega^{\omega} \cdot 2$ is not definable with parameters $\omega^{\omega}$ and 2).

As for the cofinality function, let $\tau$ be the first regular cardinal which is not definable. Let $\alpha=\tau+\tau$. All the definable $\gamma \leq \alpha$ are smaller than $\tau$ by Theorem 3.5 , and therefore $\operatorname{trunc}_{\gamma}(\alpha)$ is always either $\alpha$ or 0 for definable $\gamma$. By Theorem $4.5, \tau$ is not definable with parameter $\alpha$.

Claim 4.6 is not surprising in the case of monadic definability. But notice that each of its clauses fails with the added condition that $\alpha$ and $\beta$ are definable, by Claims 2.2 and 2.3 and Lemma 3.2.

\section{REFERENCES}

[1] J. Richard Büchi, Decision methods in the theory of ordinals, Bull. Amer. Math. Soc., vol. 71 (1965), pp. 767-770.

[2] J. Richard BüChi and Charles Zaiontz, Deterministic automata and the monadic theory of ordinals $<\omega_{2}$, Z. Math. Logik Grundlag. Math., vol. 29 (1983), no. 4, pp. 313336.

[3] Menachem Magidor, Reflecting stationary sets, J. Symbolic Logic, vol. 47 (1982), no. 4, pp. $755-771$ (1983).

[4] ITAy NeEman, Finite state automata and monadic definability of singular cardinals, To appear.

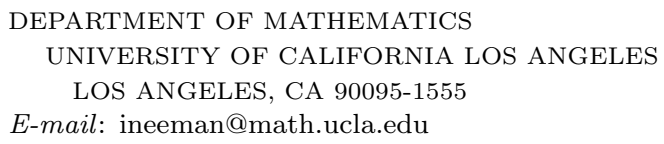

